\title{
Information Acquisition in a War of Attrition*
}

\author{
Kyungmin Kim ${ }^{\dagger}$ and Frances Zhiyun $\mathrm{Xu}^{\ddagger}$
}

April 2011

\begin{abstract}
We consider a war of attrition in which the winner is determined according to the unobservable state of nature on a stochastic deadline and players can acquire information about the state at any time during the game. We study how the players' incentive to acquire information interacts with the verifiability of the acquired information. When the information is verifiable, players only have an incentive to free ride on the opponent's information acquisition and, thus, there is excessive delay. When the information is unverifiable, an informed player obtains information rents. This provides an incentive for players to acquire information more quickly, thereby reducing delay. However, an uninformed player catches up on information acquisition so as not to be exploited by the informed player, which creates redundant duplication in information acquisition. We show that in the most natural class of equilibria the two effects cancel each other out and, thus, the players' payoffs are identical, regardless of whether information is verifiable. We also show that, in our model, the faster the deadline arrives, the longer the conflict lasts.
\end{abstract}

JEL Classification Numbers: C78, D82, D83.

Keywords : Information Acquisition; War of Attrition; Information Rent; Free Riding.

\section{Introduction}

In many economic problems, information has to be acquired, rather than exogenously given, as typically assumed in the literature. In addition, it has been shown that costly information

*We are grateful to Jacques Crémer, Johannes Hörner, Kathryn Spier, Wing Suen and seminar audiences at SHUFE for helpful comments and suggestions. All errors remain our own.

†University of Iowa, kyungmin-kim@uiowa.edu

†University of Hong Kong, zfxu@hku.hk 
acquisition may have a significant impact on the economic outcome in a variety of situations. Examples include auctions (Matthews, 1984; Persico, 2000; and Dang, 2007), bargaining (Shavell, 1994; and Dang, 2008), committee design (Li, 2001; and Persico, 2004), principalagent models (Crémer and Khalil, 1992; and Crémer, Khalil, and Rochet, 1998a, 1998b), reputation games (Ely and Välimäki, 2003; and Liu, 2010), and strategic experimentation (Bolton and Harris, 1999; and Keller, Rady, and Cripps, 2006).

When information has to be acquired, a key question is whether it can be acquired efficiently. There are two fundamental non-cooperative incentives concerning information acquisition. First, information reduces uncertainty for everyone and, therefore, has a publicgood property. Players have an incentive to free ride on others' information acquisition (the free-riding incentive). This incentive leads to underinvestment in information acquisition. Second, if acquired information remains private, then an informed player collects information rents. The incentive to obtain information rents (the information-rent incentive) leads to overinvestment in information acquisition. In most existing studies, only one of these two incentives is operative. For example, the committee-design literature and the strategicexperimentation literature exclusively focus on the free-riding problem. In bargaining and auctions, the focus typically shifts to issues related to information rents. The purpose of this paper is to demonstrate how the two fundamental non-cooperative incentives interact with each other when they are both present.

The context is a war of attrition with a deadline. In the standard war of attrition, the game ends only when at least one of the players concedes. In ours, the game exogenously terminates on a (random or deterministic) deadline, with the termination payoffs depending on the unobservable state of nature. ${ }^{1}$ When deciding whether to continue (before the arrival of the deadline), players have an incentive to learn about the state of nature: the opportunity cost of concession is larger to a player who is favored by the state and, thus, acquiring information allows a player to avoid regrettable concession (when the state is favorable) as well as unnecessary delay (when the state is unfavorable).

To focus more on economic forces, we consider the simplest form of information acquisition: a player observes the state if he incurs a positive cost. In addition, we concentrate on the case where both the deadline and information acquisition are most relevant: the deadline does not arrive too slowly, so that in the absence of information acquisition the conflict is resolved only by the arrival of the deadline, and information acquisition is not too costly, so

\footnotetext{
${ }^{1}$ In the literature on deadline effects, the deadline is often assumed to be the moment at which the potential surplus dissipates or depreciates (see, e.g., Spier (1992), Fershtman and Seidmann (1993), Ma and Manove (1993), and Ponsati (1995)). In our model, the deadline is the moment at which the winner of the game is determined according to the unobservable state. Since the deadline essentially reveals the state, we will also refer to the deadline as the "public signal."
} 
that players have a non-trivial incentive to acquire information.

We study and compare two versions of the model. ${ }^{2}$ The two cases differ with respect to whether the acquired information is verifiable. The two non-cooperative incentives regarding information acquisition are manifested differently in the two cases. When information is verifiable, as in the disclosure game, ${ }^{3}$ private information is unraveled: it is strictly dominant for an informed player to reveal favorable information and, thus, if an informed player does not disclose his private information, the opponent can infer that the state is favorable to him. Consequently, acquired information is essentially public and only the free-riding incentive is operative. When information is not verifiable, unraveling does not necessarily occur and an informed player obtains positive information rents. In other words, the weak type (who knows that the state is unfavorable to him) can always mimic the strong type and, thus, an informed player must be compensated in order to reveal his type.

In each case, there are many equilibria, as in the standard war of attrition and essentially for the same reason: players are indifferent between acquiring information and waiting at any point in time, including the beginning of the game. We fully characterize the set of all equilibria but mainly focus on the most natural class of equilibria in which no player acquires information with a positive probability at the beginning of the game. ${ }^{4}$

We show that the game ends faster when information is unverifiable, but the players' expected payoffs are identical in the two cases. As explained before, when information is verifiable, only the free-riding incentive is operative, which causes excessive delay in information acquisition. When information is not verifiable, the free-riding incentive is still operative, but is (partially) offset by the information-rent incentive. This translates into less delay with unverifiable information.

When information is unverifiable, however, there is another source of inefficiency: duplication in information acquisition. When an informed player attempts to extract information rents, an uninformed player can respond by acquiring information himself, that is, he can catch up on acquiring information to protect himself from being exploited. Such duplication never occurs with verifiable information but does occur with unverifiable information. It turns out that the positive effect from less delay exactly cancels out the negative effect from duplication. As a result, the verifiability of the acquired information significantly affects equilibrium behavior but not the players' payoffs.

We caution that the payoff equivalence does not hold if all equilibria are considered. The most efficient equilibrium in each case is the one in which one player, say player 1 ,

\footnotetext{
${ }^{2}$ A player's action of acquiring information is observable by the opponent. We discuss this assumption in Section 8.

${ }^{3}$ See Grossman (1981) and Milgrom (1981) for seminal contributions.

${ }^{4}$ See Section 6 for several reasons why such equilibria are more appealing than others.
} 
acquires information for sure at the beginning of the game. Player 1's expected payoff is the same as in the equilibrium in which no player acquires information at the beginning, but player 2's expected payoff is maximized among all the equilibria. Comparing the two cases, player 2's expected payoff is strictly higher when information is verifiable. This is because there is no delay in the most efficient equilibria (no free-riding effect), while the information-rent problem that is present only when information is unverifiable still causes excessive information acquisition.

Although our analysis is mainly theoretically motivated, our model fits into several applications. Many economic problems can be represented by wars of attrition, such as litigation (Ordover and Rubinstein, 1986), oligopolistic competition with the option to exit (Fudenberg and Tirole, 1986; Ghemawat and Nalebuff, 1985; and Kreps and Wilson, 1982), patent races (Fudenberg, Gilbert, Stiglitz, and Tirole, 1983), and public-good provision (Bliss and Nalebuff, 1984; and Bulow and Klemperer, 1999). The conflicts are often resolved by an exogenous force. In a legal conflict, the trial eventually determines whether the defendant is guilty. In a competition over a technology standard, the government may intervene or public signals that prove clear superiority of one technology over the other may arrive. Our model captures in a simple way the problems facing agents in such situations.

Our analysis provides novel insights for such applications. Our main result suggests that whether the government (or society) should require agents involved in a conflict to disclose their information or provide a verification service for their information depends on the objective of the government (or society). If the objective is to maximize the agents' joint surplus, as in civil charges, a mandatory disclosure rule or a verification service would not help. If the objective is, in addition, to have the right agent win, as in criminal charges or technology standard competitions, then a mandatory disclosure rule and a verification service are marginally useful. ${ }^{5}$ If the objective also includes ending the conflict as quickly as possible, as in the public-good-provision problem, then a mandatory disclosure rule should not be imposed and a verification service should not be provided.

Our model can also be used to address other policy issues in such applications. For example, in a legal conflict, the government may regulate attorney fees or impose a stricter deadline. In a technology standard competition, the government may intervene to control the costs of the participants or invest in research to evaluate the merits of each technology. Our results provide simple answers to these questions.

One particularly interesting result is that the shorter the deadline is, the longer the conflict lasts (regardless of whether the information is verifiable). Increasing the arrival rate of the deadline/public signals directly speeds up the resolution of a conflict. However, in our

\footnotetext{
${ }^{5}$ With unverifiable information, a wrong winner may be selected, but the probability is negligible.
} 
model, where players can acquire information about the state, such a rate increase has the indirect effect of crowding out the players' incentive to acquire information. The players' incentive to free-ride on information acquisition comes from the possibility that the public signal may arrive soon or that the opponent may acquire information first, both of which help them avoid the information-acquisition costs. Therefore, as the arrival rate of public signals increases, players are less willing to acquire information. In our model, this indirect effect is at least twice as large as the direct effect ${ }^{6}$ and, thus, shortening deadlines delays the resolution of a conflict.

\section{Related Literature}

The literature on the war of attrition and its applications is too large to summarize here. Let us introduce only a few seminal contributions. The game is first proposed by Maynard Smith (1974). The analysis of the game with complete information is generalized by Bishop and Cannings (1978) and, subsequently, by Hendricks, Weiss, and Wilson (1988). Various versions of the game with incomplete information are also studied (for example, Bishop, Cannings, Maynard Smith (1978), Riley (1980), Milgrom and Weber (1985), Nalebuff and Riley (1985), and Ponsati and Sákovics (1995)). Bulow and Klemperer (1999) generalize the game into the case with $N+k$ players and $N$ winners. Our model is unique in that the game begins with complete information but turns into a game with incomplete information once a player acquires information, that is, asymmetric information is endogenized.

The closest papers to ours are those by Ordover and Rubinstein (1986) and Morath (2010). Ordover and Rubinstein (1986) study a war of attrition between an informed player and an uninformed player. As in our paper, the information of the informed player is about who wins at the (deterministic) deadline. The crucial difference is that, in our model, the information is endogenous and both players can learn about the state at any point in time, while in Ordover and Rubinstein (1986), the information is exogenous and the uninformed player does not have the ability to acquire information. Our analysis reveals that both the endogeneity of information and the uninformed player's access to the informationacquisition technology have significant effects on the equilibrium outcome. Morath (2010) studies the model in which players can acquire information about the cost of fighting at (and only at) the beginning of the game. He is mainly interested in the strategic value of (not) acquiring information and, therefore, considers the case where the players' values of losing

\footnotetext{
${ }^{6}$ From an individual player's viewpoint, the arrival of the public signal and the opponent's information acquisition are perfect substitutes. Therefore, for both players to remain indifferent after the increase in the arrival rate, each player's concession rate must decrease by at least as much as the increase in the arrival rate. See Section 7 for more-detailed arguments.
} 
are independent and information acquisition is costless.

A few papers study the situations in which the free-riding and information-rent incentives interact. Grossman and Stiglitz (1980) study the competitive market in which investors can acquire information about the return of a risky asset. Investors, if they become informed, can receive arbitrage profits (information rents). However, the uninformed players can infer about the information of the informed investors through competitive prices and, thus, investors also have a free-riding incentive. They show that this conflict between the two incentives leads to competitive prices only partially reflecting the information of the informed investors. Jansen (2008) considers the oligopoly setting in which firms may acquire information about the demand curve. If firms must disclose their acquired information, then, due to the free-riding incentive, too little information is acquired. If firms cannot credibly disclose their acquired information, then, due to the information-rent incentive, too much information is acquired. If firms can choose whether or not to disclose, then, due to the conflict between the two incentives, firms' expected payoffs may be higher (and lower) than under no disclosure or full disclosure.

The remainder of this paper is organized as follows. Section 2 presents and analyzes the benchmark model in which information acquisition is not allowed. Section 3 presents the model with information acquisition. Section 4 and Section 5 analyze the case where acquired information is verifiable and the case where it is not, respectively. Section 6 highlights the role of the verifiability of the information by comparing the two models. Section 7 demonstrates that shortening the deadline delays the resolution of a conflict. Section 8 concludes by discussing two particularly interesting extensions.

\section{War of Attrition with Deadlines}

\subsection{Setup}

Our underlying model is the standard war of attrition. There are two players, player 1 and player 2. Each player chooses the time to concede, $t_{i} \in \mathcal{R}_{+}$. If player $j$ concedes first $\left(t_{j}<t_{i}\right)$, then player $i$ receives utility $e^{-r t_{j}} h$, while player $j$ receives utility $e^{-r t_{j}} l$, where $h>l>0$. In other words, at time $\min \left\{t_{1}, t_{2}\right\}$, the loser (who conceded) receives $l$ and the winner receives $h$, and the common discount rate is $r>0$. For simplicity, we assume that if $t_{i}=t_{j}$, both players obtain $e^{-r t_{i}} l .^{7}$ Let $d$ denote the undiscounted reward to the winner, that is, $d \equiv h-l$.

\footnotetext{
${ }^{7}$ This is without loss of generality, as the event that both players concede simultaneously occurs with probability 0 in all of the cases we consider.
} 
Unlike the standard game, our game can also terminate exogenously. More specifically, there is an underlying state of nature, $\omega \in\{1,2\}$. The state $\omega$ is initially unknown, but a public signal that reveals the state arrives according to a Poisson rate $\lambda>0$. Upon the arrival of the signal, the game ends and player $i$ receives utility $h(l)$ if $\omega=i(j)$. It is commonly known that $\omega=1$ with probability $p_{1} \in[0.5,1)$ and $\omega=2$ with probability $p_{2}=1-p_{1}$.

Within each time interval $[t, t+d t)$, the timing of the game is as follows: a public signal arrives with probability $1-e^{-\lambda d t}$. If it arrives, then the game ends according to the revealed state. If not, the players simultaneously decide whether or not to concede. Although we study a continuous-time model, the model can be interpreted as the limit of the discrete-time models in which players move only at $t=\Delta, 2 \Delta, \ldots$. We use this discrete-time version to clarify some of our results.

\subsection{Characterization}

Let a distribution function $G_{i}: \mathcal{R}_{+} \rightarrow[0,1]$ represent player $i$ 's concession strategy, where $G_{i}(t)$ is the cumulative probability that player $i$ concedes by time $t$. By standard argument, $G_{i}$ has no atom in its interior. In addition, unless the equilibrium is degenerate (a player concedes at the beginning of the game), the supports of equilibrium $G_{1}$ and $G_{2}$ are common and take an interval starting from time 0 . Let $g_{i}$ denote the density of $G_{i}$ over the interior of its support.

As in the standard war of attrition, at each $t$ in the interior of the support, players must be indifferent between conceding and waiting an instant more. Therefore, for each $i=1,2$ and $j \neq i$,

$$
r l=\frac{g_{j}(t)}{1-G_{j}(t)} d+\lambda p_{i} d .
$$

The left-hand side is player $i$ 's marginal cost of waiting an instant more, while the right-hand side is the corresponding marginal benefit. ${ }^{8}$ Player $i$ receives $l$ if he concedes. His marginal

${ }^{8}$ The analogous indifference condition in the discrete-time model is

$$
l=\frac{G_{j}(t)-G_{j}(t-\Delta)}{1-G_{j}(t-\Delta)} h+\frac{1-G_{j}(t)}{1-G_{j}(t-\Delta)} e^{-r \Delta}\left(\left(1-e^{-\lambda \Delta}\right)\left(l+p_{i} d\right)+e^{-\lambda \Delta} l\right) .
$$

The left-hand side is player $i$ 's payoff by conceding, while the right-hand side is his payoff by waiting one more period. If player $i$ does not concede, then player $j$ concedes with probability $\frac{G_{j}(t)-G_{j}(t-\Delta)}{1-G_{j}(t-\Delta)}$ in this period. With the complementary probability, the game moves to the next period. In the next period, a public signal arrives with probability $1-e^{-\lambda \Delta}$, in which case player $i$ receives $h$ with probability $p_{i}$ and $l$ with probability $1-p_{i}$. If a signal does not arrive, for a small enough $\Delta$, player $i$ is again indifferent between conceding and waiting one more period and, thus, player $i$ 's expected payoff is $l$. It is straightforward to show that this discrete-time condition converges to the continuous-time condition as $\Delta$ tends to zero. 
cost of staying an instant more is the discounting cost of collecting the payoff an instant later. If player $i$ wait an instant more, he obtains an additional payoff $d$ under the following two contingencies: (1) player $j$ concedes before $t+d t$, whose arrival rate is $\frac{g_{j}(t)}{1-G_{j}(t)} ;(2)$ a public signal arrives and the state is revealed to be favorable to player $i$ (that is, $\omega=i$ ). The arrival rate of the signal is $\lambda$ and the probability that $\omega=i$ is $p_{i}$.

If $r l<\lambda p_{i} d$, then the marginal benefit is always larger than the marginal cost. Therefore, player $i$ never concedes. If $r l \geq \lambda p_{i} d$, then the indifference condition has a closed-form solution:

$$
G_{j}(t)=1-\left(1-G_{j}(0)\right) \exp \left(-\left(\frac{r l-\lambda p_{i} d}{d}\right) t\right),
$$

where $G_{j}(0) \in[0,1]$ is unknown.

There are essentially three cases to consider. If $r l<\lambda p_{2} d$, then no player is willing to concede. In this case, it is the unique equilibrium that both players wait for a public signal forever. If $\lambda p_{2} d<r l<\lambda p_{1} d$, then player 1 never concedes and, given player 1's strategy, player 2 strictly prefers conceding immediately. It is the unique equilibrium that player 2 concedes immediately.

If $r l>\lambda p_{1} d$, then both $G_{1}$ and $G_{2}$ are well-defined. One restriction for the two unknowns, $G_{1}(0)$ and $G_{2}(0)$, is that at least one of them must be equal to 0 . This is because if player $i$ concedes with a positive probability at time 0 , then player $j$ strictly prefers waiting an instant more to conceding immediately. There is no further restriction on $G_{1}(0)$ and $G_{2}(0)$ and, therefore, $G_{i}(0)$ can take any value in $[0,1]$ as long as $G_{j}(0)=0$.

In the third parameter case, where $r l>\lambda p_{1} d$, as is familiar in the war-of-attrition literature, there are also degenerate equilibria: one player does not concede forever or until after a sufficiently long time, and the opponent concedes immediately. Such equilibria are essentially irrelevant, because their equilibrium outcomes coincide with those in which $G_{i}(0)=1$ for some $i$. For clarity of exposition, we ignore all such degenerate equilibria throughout the paper.

The following proposition summarizes the findings for the model without information acquisition.

Proposition 1 (i) If $r l<\lambda p_{2} d$, then there is a unique equilibrium in which both players wait for a public signal forever. Player $i$ 's expected payoff is $\frac{\lambda}{r+\lambda}\left(l+p_{i} d\right)$.

(ii) If $\lambda p_{2} d<r l \leq \lambda p_{1} d$, then it is the unique equilibrium outcome that player 2 concedes immediately. Player 2 obtains $l$, while player 1 obtains $h^{9}{ }^{9}$

\footnotetext{
${ }^{9}$ If $\lambda p_{2} d=r l \leq \lambda p_{1} d$, then there is a continuum of equilibria. If $r l<\lambda p_{1} d$, then for any $\alpha \in[0,1]$, it is an equilibrium that player 2 concedes with probability $\alpha$ at date 0 and never concedes with the complementary probability, and player 1 never concedes. Player 2's expected payoff is always $l=\frac{\lambda}{r+\lambda}\left(l+p_{2} d\right)$, while player
} 
(iii) If $r l>\lambda p_{1} d$, there is a continuum of equilibria. For any $G_{1}(0), G_{2}(0) \in[0,1]$ such that $G_{1}(0) G_{2}(0)=0$, it is an equilibrium that player $i$ concedes according to distribution

function $G_{i}(t)=1-\left(1-G_{i}(0)\right) \exp \left(-\left(\frac{r l-\lambda p_{j} d}{d}\right) t\right)$ for both $i=1,2$. Each player can obtain any expected payoff in $[l, h]$, provided that the opponent receives $l$.

Intuitively, if a public signal arrives sufficiently fast ( $\lambda$ is high), players are sufficiently patient ( $r$ is small), or the winning reward is sufficiently large ( $d$ is large), as in Case (i), players are unwilling to concede and, therefore, wait forever. In the opposite case, as in Case (iii), public signals are essentially irrelevant and, thus, the game is almost identical to the standard war of attrition. In the asymmetric case $\left(p_{1}>p_{2}\right)$, there is an intermediate case (Case (ii)): a public signal arrives fast enough, so that player 1 is willing to wait, but not too fast, so that player 2 does not want to bear delay costs. In that case, player 2 concedes immediately and player 1 obtains the highest possible payoff.

\section{The Model with Information Acquisition}

The players have an incentive to learn about the state $\omega$ in order to avoid regrettable concessions and unnecessary delay. In this section, we allow players to acquire information about $\omega$. Information acquisition is costly: each player must incur a cost $c>0$ in order to observe the state $\omega$.

Within each time interval $[t, t+d t)$, the timing of the game is as follows:

1. Signal stage: a public signal arrives with probability $1-e^{-\lambda d t}$. If it arrives, then the game terminates according to the state.

2. Information-acquisition stage: Players simultaneously decide whether or not to acquire information. Players observe whether the opponent has acquired information.

3. Disclosure stage: If acquired information is verifiable, then the player who acquired information can disclose his information. Otherwise, this stage is skipped.

4. Concession stage: Players simultaneously decide whether or not to concede.

We focus on the case where both deadline and information acquisition are most relevant. Formally, we make use of the following two assumptions.

\section{Assumption 1}

$$
\lambda p_{i} d>r l, i=1,2 .
$$

1 can obtain any utility between $[l, h]$. If $r l=\lambda p_{1} d$, the roles of the players can be switched and, thus, both players can obtain any expected payoff in $[l, h]$, provided that the opponent obtains $l$. 
This assumption states that a public signal arrives fast enough ( $\lambda$ is high), the reward of winning is large enough ( $d$ is large), or the players are patient enough ( $r$ is small), so that both players are unwilling to forgo the opportunity to win the game. Under this assumption, if no player acquires information, by Proposition 1 (Case (i)), both players wait forever and the game ends only upon the arrival of a public signal.

\section{Assumption 2}

$$
c<\frac{r l}{\lambda}
$$

To understand this assumption, suppose that no player would acquire information and both players would wait forever. If a player believes that the state is favorable to him with probability $p$, then his expected payoff is $\frac{\lambda}{r+\lambda}(l+p d)$. Now suppose the player acquires information and the game terminates according to the true state. In this case, the player's expected payoff is $-c+l+p d$. The assumption states that the latter payoff is strictly larger than the former, as long as the player does not strictly prefer conceding immediately to acquiring information, that is, $-c+l+p d>\frac{\lambda}{r+\lambda}(l+p d)$ for any $p$ such that $-c+l+p d \geq l$. Intuitively, this assumption guarantees that the cost of information acquisition is not too large, so players have a non-trivial incentive to acquire information.

For notational simplicity, we will refer to a player who has acquired information and found that the state is favorable (unfavorable) to him as the "strong" ("weak") type.

\section{Information Acquisition with Verifiable Information}

This section considers the case where players can verify acquired information.

We begin with three immediate results. First, the game ends immediately once at least one player acquires information. This is because it is a dominant strategy for the strong type to disclose the acquired information. If an informed player does not disclose the information, then the opponent would know that the state is favorable to him and, therefore, would never concede. The weak informed player would then immediately concede. Second, the game endogenously concludes only when at least one player acquires information. This is because, by Assumption 1, if no player acquires information, then both players would wait forever. Third, if player $j$ never acquires information, then player $i$ acquires information immediately. This is because, by Assumptions 1 and 2, player $i$ 's expected payoff by acquiring information $-c+l+p_{i} d$ is strictly larger than his expected payoff by waiting forever $\frac{\lambda}{r+\lambda}\left(l+p_{i} d\right)$.

The above results imply that the only strategic problem is who acquires the information. Since the information is verifiable, information unraveling prevents players from extracting any information rents. Therefore, only the free-riding incentive is present and the game is 
essentially a war of attrition. The difference from the standard war of attrition is that now it is not about who concedes first, but who acquires the information first.

For a formal description of the equilibrium, let a distribution function $F_{i}: \mathcal{R}_{+} \rightarrow[0,1]$ represent player $i$ 's information-acquisition strategy, where $F_{i}(t)$ is the cumulative probability that player $i$ acquires information by time $t$. By standard argument, $F_{i}$ has no atom in its interior. Let $f_{i}$ denote the density of $F_{i}$ over the interior of its support. As familiar, if $t$ is in the interior of the support of $F_{i}$, player $i$ must be indifferent between acquiring information and waiting an instant more. Therefore,

$$
r\left(-c+l+p_{i} d\right)=\left(\lambda+\frac{f_{j}(t)}{1-F_{j}(t)}\right) c .
$$

The left-hand side is player $i$ 's marginal cost of delaying information acquisition an instant, while the right-hand side is the corresponding marginal benefit. ${ }^{10}$ The marginal cost is his discounting cost of collecting the payoff by acquiring information, $-c+l+p_{i} d$, an instant later. The marginal benefit comes from the fact that during that instant, a public signal may arrive, at an arrival rate of $\lambda$, or the opponent may acquire information, at an arrival rate of $\frac{f_{j}(t)}{1-F_{j}(t)}$. Under both contingencies, player $i$ avoids the information-acquisition cost $c$.

Solving the first-order ordinary differential equation,

$$
F_{j}(t)=1-\left(1-F_{j}(0)\right) \exp \left(-\left(r \frac{-c+l+p_{i} d}{c}-\lambda\right) t\right)
$$

where $F_{j} \in[0,1]$ is unknown. Under Assumptions 1 and 2, the function is always welldefined. One restriction for the two unknowns $F_{1}(0)$ and $F_{2}(0)$ is that at least one of them must be equal to zero, that is, $F_{1}(0) F_{2}(0)=0$. Similarly to the standard argument, this is because if a player acquires information with a positive probability at date 0 , then the other player strictly prefers waiting an instant. As in Section 2, there is no further restriction for the two unknowns. Therefore, $F_{1}(0)$ and $F_{2}(0)$ can take any values in $[0,1]$ as long as $F_{1}(0) F_{2}(0)=0$.

The following proposition summarizes the findings. Given the characterization above, the proof is straightforward and thus omitted.

Proposition 2 When acquired information is verifiable, there is a continuum of equilibria: for each $F_{1}(0), F_{2}(0) \in[0,1]$ such that $F_{1}(0) F_{2}(0)=0$, there is an equilibrium in

\footnotetext{
${ }^{10}$ The discrete-time analog to this equation is

$-c+l+p_{i} d=\frac{F_{j}(t)-F_{j}(t-\Delta)}{1-F_{j}(t-\Delta)}\left(l+p_{i} d\right)+\frac{1-F_{j}(t)}{1-F_{j}(t-\Delta)} e^{-r \Delta}\left(\left(1-e^{-\lambda \Delta}\right)\left(l+p_{i} d\right)+e^{-\lambda \Delta}\left(-c+l+p_{i} d\right)\right)$.
} 
which player $i$ acquires information according to the distribution function $F_{i}(t)=1-(1-$

$\left.F_{i}(0)\right) \exp \left(-\left(r \frac{-c+l+p_{j} d}{c}-\lambda\right) t\right)$ for both $i=1,2$. The set of the players' expected payoffs is given by

$$
\left\{\left(v_{1}, v_{2}\right): v_{i} \in\left[-c+l+p_{i} d, l+p_{i} d\right] \text {, and }\left(v_{1}-\left(-c+l+p_{1} d\right)\right)\left(v_{2}-\left(-c+l+p_{2} d\right)\right)=0\right\} \text {, }
$$

that is, player $i$ can achieve any payoff in $\left[-c+l+p_{i} d, l+p_{i} d\right]$ as long as the opponent receives $-c+l+p_{j} d$.

\section{Information Acquisition with Unverifiable Informa- tion}

This section studies the case where acquired information is not verifiable. The difference from the previous section is that there is no disclosure stage (or the disclosed information is not credible).

We first characterize the outcome of a game in which one player is informed about the state and the other is not. This is the subgame immediately following one player's information acquisition. We use the outcome of this game to characterize the equilibrium in the original game.

\subsection{Subgame in which one player is informed}

This game is similar to that of Ordover and Rubinstein (1986). The difference lies in the uninformed player's strategy set. In Ordover and Rubinstein (1986), the uninformed player can choose only whether or not to concede, while in our game the uninformed player can acquire information. We show that this difference makes the equilibrium dynamics of our game significantly different from those of Ordover and Rubinstein (1986).

For expositional clarity, consider the discrete-time version of the model. We begin with two results concerning the equilibrium behavior of the weak informed player in the first period of the subgame. First, the weak informed player must concede with a positive probability. Otherwise, the uninformed player would either acquire information or wait for a public signal, and then, due to discounting, the weak informed player would get strictly less than $l$. Second, the weak informed player does not concede with probability 1 . If he concedes with probability 1 , then in the next period the uninformed player would believe that he is facing a strong type with probability 1 and, therefore, concedes without acquiring information for sure. But the weak informed player would then strictly prefer waiting to conceding in the 
first period, which is a contradiction.

The fact that the weak informed player stays with a positive probability but still obtains $l$ implies the following two results regarding the uninformed player's behavior in the second period. First, the uninformed player must concede without acquiring information with a certain probability. Otherwise, the weak informed player would receive only $l$ in the second period, regardless of whether the uninformed player acquires information or waits forever. This is a contradiction because he could have received the same payoff in the first period. Second, the concession probability of the uninformed player must be small enough. Otherwise, the weak informed player would strictly prefer waiting to conceding in the first period.

In equilibrium, the weak informed player randomizes between conceding and waiting in the first period. The uninformed player randomizes between acquiring information and conceding in the second period. They do so with just enough probabilities so that both the weak informed player and the uninformed player are indifferent between their two actions. The game ends for sure in the second period.

Formally, let $\alpha$ be the probability that the weak informed player concedes in the first period. Also, let $\beta$ be the probability that the uninformed player acquires information in the second period. The following two conditions must be satisfied:

1. Weak informed player $i$ 's indifference:

$$
l=e^{-r \Delta}\left(\left(1-e^{-\lambda \Delta}\right) l+e^{-\lambda \Delta}(\beta l+(1-\beta) h)\right) .
$$

If the weak informed player does not concede, then in the next period a public signal arrives with probability $1-e^{-\lambda \Delta}$, in which case the player receives $l$. Conditional on the event that a signal does not arrive, the uninformed player acquires information with probability $\beta$ and concedes with the complementary probability. The weak informed player receives $l$ and $h$ in each event. Solving this equation,

$$
\beta=1-\frac{\left(1-e^{-r \Delta}\right) l}{e^{-(r+\lambda) \Delta} d} .
$$

This value is well-defined as long as $\Delta$ is sufficiently small.

2. Uninformed player $j$ 's indifference:

$$
-c+l+\frac{p_{j}(1-\alpha)}{p_{i}+p_{j}(1-\alpha)} d=l .
$$

The left-hand side is player $j$ 's expected payoff by acquiring information in the second 
period. Conditional on the event that player $i$ did not concede in the first period, by Bayes' rule, player $j$ 's belief over the state is $\frac{p_{j}(1-\alpha)}{p_{i}+p_{j}(1-\alpha)}$. The right-hand side is his payoff by conceding. Solving the equation,

$$
\alpha=1-\frac{p_{i} c}{p_{j}(d-c)}
$$

This probability is also well-defined because, by Assumptions 1 and 2,

$$
c<\frac{r}{\lambda+r}\left(l+p_{j} d\right)<\frac{r}{\lambda+r}\left(\frac{\lambda p_{j} d}{r}+p_{j} d\right)=p_{j} d .
$$

Probability $\alpha$ is independent of $\Delta$, while $\beta$ approaches one as $\Delta$ tends to zero. Therefore, in the equilibrium of the continuous-time model, the weak informed player immediately concedes with probability $\alpha$. If the informed player does not concede, then an instant later the uninformed player acquires information with probability 1 . One may wonder why the weak informed player does not prefer conceding immediately to waiting, given that the uninformed player acquires information with probability 1 in the next period and, thus, the weak informed player cannot obtain more than $l$ even if he waits. This is because in continuous time the cost of waiting an instant is negligible. In compensating the weak informed player's cost of waiting an instant, it is enough for the uninformed player to concede with negligible probability. In turn, this is why the uninformed player must remain indifferent between acquiring information and conceding, even if in equilibrium he acquires information with probability 1.

Proposition 3 (Subgame outcome) In the subgame in which only player $i$ is informed about the state, there is a unique equilibrium. In equilibrium, weak player $i$ concedes with probability $\frac{p_{j} d-c}{p_{j}(d-c)}$. If informed player $i$ does not concede, then uninformed player $j$ acquires information and terminates the game. Strong (weak) player $i$ obtains $h(l)$. Player $j$ 's expected payoff is $l+\frac{p_{j} d-c}{d-c} d$.

Proof. See the Appendix.

Let us quantify the amount of information rents and the value of the uninformed player's information-acquisition opportunity.

Information rents If player $i$ is informed but his opponent is not, then his expected payoff is $l+p_{i} d$. If the opponent is informed but player $i$ is not, then his expected payoff is $l+\frac{p_{i} d-c}{d-c} d$. Therefore, the additional payoff player $i$ collects by acquiring information first 
amounts to

$$
\left(l+p_{i} d\right)-\left(l+\frac{p_{i} d-c}{d-c} d\right)=\frac{\left(1-p_{i}\right) c d}{d-c} .
$$

The value of the opportunity to acquire information Suppose the uninformed player cannot acquire information. The game is then essentially the stochastic deadline version of Ordover and Rubinstein (1986). In this game, the weak informed player concedes with a positive probability in the first period. From the second period on, he strictly prefers waiting to conceding and, therefore, never concedes. The uninformed player strictly prefers waiting to conceding in the first period and is indifferent between conceding and waiting from the second period forward. Therefore, in equilibrium, the weak informed player concedes only in the first period, while the uninformed player gradually concedes starting from the second period.

Let $\alpha$ be the probability that weak informed player $i$ concedes in the first period. Also, let a distribution function $G_{j}: \mathcal{R}_{+} \rightarrow[0,1]$ represent uninformed player $j$ 's concession strategy. Then the following two conditions must be satisfied:

1. Uninformed player $j$ 's indifference: Uninformed player $j$ is indifferent between conceding and waiting at each point in time, except at the beginning of the game. Therefore,

$$
r l=\lambda \frac{p_{j}(1-\alpha)}{p_{i}+p_{j}(1-\alpha)} d
$$

The left-hand side is uninformed player $j$ 's marginal cost of waiting an instant, while the right-hand side is the corresponding marginal benefit. The latter only comes from the possibility of the arrival of a public signal, because informed player $i$ never concedes, regardless of whether he is strong or weak. Solving the equation,

$$
\alpha=\frac{p_{j} \lambda d-r l}{p_{j}(\lambda d-r l)} .
$$

2. Weak player $i$ 's expected payoff: At each point in time, including the beginning of the game, weak player $i$ expects to receive $l$ if the game continues, that is, if neither player concedes in the period. Therefore, in the limit as $\Delta$ tends to zero,

$$
r l=\frac{g_{j}(t)}{1-G_{j}(t)} d .^{11}
$$

${ }^{11}$ The discrete-time analogue to this equation is

$$
l=e^{-r \Delta}\left(\left(1-e^{-\lambda \Delta}\right) l+e^{-\lambda \Delta} \frac{G(t+\Delta)-G(t)}{1-G(t)} h+e^{-\lambda \Delta} \frac{1-G(t+\Delta)}{1-G(t)} l\right) .
$$


If weak player $i$ does not concede, uninformed player $j$ may concede, at an arrival rate of $\frac{g_{j}(t)}{1-G_{j}(t)}$. Unlike the previous cases, the arrival of a public signal does not contribute to the marginal benefit, because weak player $i$ definitely loses in that event. Solving this equation,

$$
G_{j}(t)=1-\exp \left(-\frac{r l}{d} t\right)
$$

Notice that $G_{j}(0)=0$, because if $G_{j}(0)>0$, then weak player $i$ would strictly prefer waiting an instant to conceding at the beginning of the game.

In this game, uninformed player $j$ obtains

$$
l+p_{j} \alpha d=l+\frac{p_{j} \lambda d-r l}{\lambda d-r l} d .
$$

The value of the information-acquisition opportunity to the uninformed player is the difference between his payoff in Proposition 3 and this payoff, which amounts to

$$
\left(l+\frac{p_{j} d-c}{d-c} d\right)-\left(l+\frac{p_{j} \lambda d-r l}{\lambda d-r l} d\right)=\frac{\left(1-p_{j}\right) d(r l-\lambda c)}{(d-c)(\lambda d-r l)} d .
$$

Under Assumption 2, this value is always positive. This result shows that the option to acquire information is indeed valuable to the uninformed player.

Weak informed player $i$ again obtains $l$, while strong informed player $i$ 's expected payoff is

$$
\int_{0}^{\infty} e^{-r t} h d\left(1-e^{-\lambda t}\left(1-G_{j}(t)\right)\right)=\frac{\lambda+\frac{r l}{d}}{r+\lambda+\frac{r l}{d}} h .
$$

The latter payoff is strictly smaller than the corresponding payoff, $h$, in Proposition 3 . This result is somewhat surprising because the opponent (uninformed player $j$ ) is in a stronger position and receives a higher payoff when he can acquire information. The driving force for this result is that the role of the uninformed player's information acquisition is mainly to reduce unnecessary delay, which applies to the informed player as well as the uninformed player.

If the game moves to the next period, a public signal arrives with probability $1-e^{-\lambda \Delta}$, in which case weak player $i$ receives $l$. Weak player $i$ does not concede, unless it is the first period. He receives $h$ if uninformed player $j$ concedes. Otherwise, his contiuation payoff is equal to $l$. Note that weak player $i$ strictly prefers waiting to conceding, because his expected payoff by waiting $\frac{G(t+\Delta)-G(t)}{1-G(t)} h+\frac{1-G(t+\Delta)}{1-G(t)} l$ is strictly larger than $l$. 


\subsection{The (original) game in which both players are uninformed}

Now we consider the original game in which both players start out being uninformed about the state.

As with verifiable information, the game ends endogenously only when at least one player acquires information. This is because of Assumption 2 and Proposition 3: if player $i$ never acquires information, then player $j$ strictly prefers acquiring information to waiting because his expected payoff by acquiring information $-c+l+p_{i} d$ (Proposition 3 ) is strictly larger than his expected payoff by not acquiring information (Assumption 2).

In addition, players prefer for the opponent to acquire information first. Although an informed player receives information rents, the cost of information acquisition always outweighs information rents:

$$
c-\frac{\left(1-p_{i}\right) c d}{d-c}=\frac{p_{i} d-c}{d-c} c>0 .
$$

Intuitively, player $i$ obtains $l+p_{i} d$ if he is informed about the state, while his expected payoff when he is uninformed is bounded below by $-c+l+p_{i} d$. Therefore, the information rent cannot be larger than the information acquisition cost $c$.

The two results imply that the game is again a war of attrition regarding who acquires information first. The difference from the verifiable case is in the players' payoffs after one of the players acquires information. The informed player obtains the same payoff as in the verifiable case, but the opponent receives a strictly lower payoff. The latter occurs because in equilibrium the uninformed player also acquires information with a positive probability.

For a direct comparison, let us use the same notations for the players' informationacquisition strategies as in the verifiable case. As usual, if $t$ is in the interior of the support of $F_{i}$, then player $i$ must be indifferent between acquiring information and delaying it for an instant. Therefore,

$$
r\left(-c+l+p_{i} d\right)=\left(\lambda+\frac{f_{j}(t)}{1-F_{j}(t)} \frac{p_{i} d-c}{d-c}\right) c .
$$

The left-hand side is player $i$ 's marginal cost of acquiring information an instant later, while the right-hand side is the corresponding marginal benefit. If player $i$ does not acquire information right now, then a public signal may arrive, at an arrival rate of $\lambda$, or the opponent

may acquire information, at an arrival rate of $\frac{f_{j}(t)}{1-F_{j}(t)}$. In the latter case, player $i$ avoids the information-acquisition cost $c$ but loses information rents, $\frac{\left(1-p_{i}\right) d}{d-c} c$.

The solution to this first-order ordinary differential equation is

$$
F_{j}(t)=1-\left(1-F_{j}(0)\right) \exp \left(-\frac{d-c}{p_{i} d-c}\left(r \frac{-c+l+p_{i} d}{c}-\lambda\right) t\right) .
$$


Again, as usual, $F_{1}(0)$ and $F_{2}(0)$ can take any values in $[0,1]$ as long as at least one of them is equal to zero. Therefore, there is a continuum of equilibria.

Proposition 4 When acquired information is not verifiable, there is a continuum of equilibria: for each $F_{1}(0), F_{2}(0) \in[0,1]$ such that $F_{1}(0) F_{2}(0)=0$, there is an equilibrium in which player $i$ acquires information according to the distribution function $F_{i}(t)=1-(1-$ $\left.F_{i}(0)\right) \exp \left(-\frac{d-c}{p_{j} d-c}\left(r \frac{-c+l+p_{j} d}{c}-\lambda\right) t\right)$ for both $i=1,2$. The set of players' payoffs is given by

$\left\{\left(v_{1}, v_{2}\right): v_{i} \in\left[-c+l+p_{i} d, l+\frac{p_{j} d-c}{d-c} d\right]\right.$, and $\left.\left(v_{1}-\left(-c+l+p_{1} d\right)\right)\left(v_{2}-\left(-c+l+p_{2} d\right)\right)=0\right\}$,

that is, player $i$ can achieve any payoff in $\left[-c+l+p_{i} d, l+\frac{p_{j} d-c}{d-c} d\right]$, as long as the opponent receives $-c+l+p_{j} d$.

\section{The Role of Information Verifiability}

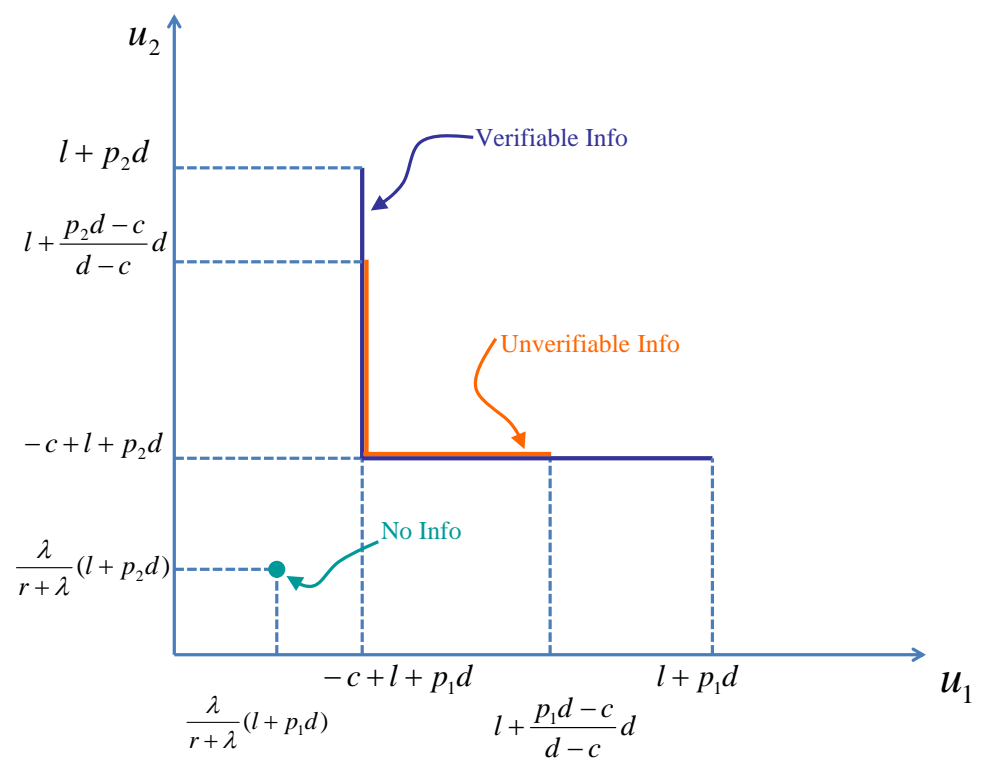

Figure 1: The sets of equilibrium payoffs

As shown in Figure 1, some payoff vectors are attainable only in the verifiable case. They are obtained in the equilibria in which a player acquires information with a sufficiently high probability at the beginning of the game and, thus, the delay in information acquisition is rather small. The payoff difference stems from the difference in equilibrium behavior after one player acquires information. In the verifiable case, the game concludes immediately 
without any further distortions. The game also terminates immediately in the unverifiable case. However, in equilibrium, the uninformed player acquires information with a positive probability and, therefore, obtains a strictly smaller payoff than in the verifiable case.

To facilitate the comparison, we focus on the equilibria in which no player acquires information with a positive probability at the beginning of the game. There are at least four reasons why such equilibria are more appealing than others. First, in the symmetric case, it is the unique symmetric equilibrium. Second, the war-of-attrition literature has focused on the same class of equilibria and, thus, we can directly place our results in the literature. Third, they are the only equilibria that do not require coordination between the players regarding who moves first. ${ }^{12}$ Last, players want the opponent to acquire information first. Therefore, if both players think that the opponent would acquire information at the beginning of the game with some probability, then such equilibria will be uniquely selected.

In such equilibria, players obtain the same payoffs regardless of whether the information is verifiable. Their payoffs coincide with the lower bounds of the payoff ranges. There are, however, two important differences.

First, the lower bounds are essentially exogenously given in the verifiable case, while they are endogenously determined in the unverifiable case. In the former case, acquired information is public, and the lower bounds are simply derived from the public nature of the information. They are the lower bounds even if we consider all the possible strategies of the players, not just the equilibrium ones. In the latter case, there is no a priori reason for the game to end immediately after a player acquires information. It is only in equilibrium that the game concludes immediately, because in equilibrium the uninformed player also acquires information immediately after.

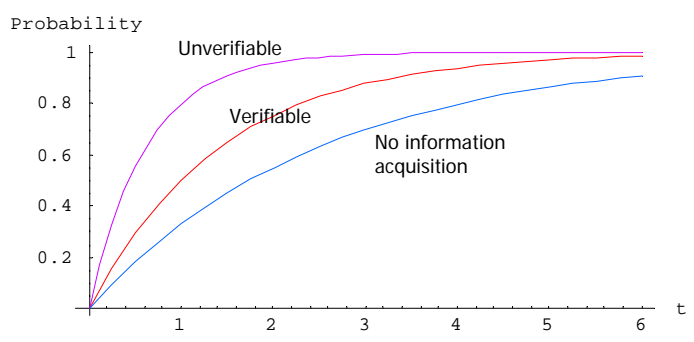

Figure 2: The cumulative probabilities that the game ends by time $t$.

Second, although the equilibrium strategies exhibit similar qualitative properties, they are quantitatively different. In particular, the game ends faster with unverifiable information. This comparison is in fact valid for any equilibria that share the same initial probabilities,

\footnotetext{
${ }^{12}$ See, for example, Levin and Smith (1994) and Burdett, Shi, and Wright (2001) for the uses of the same restriction with the same motivation in different contexts.
} 
$F_{1}(0)$ and $F_{2}(0)$. See Figure 2 for a graphical representation of how quickly the game ends for the case where $F_{1}(0)=F_{2}(0)=0$.

Proposition 5 Fix $F_{1}(0)$ and $F_{2}(0)$ and let $S_{V}(t)$ and $S_{U}(t)$ be the corresponding cumulative probabilities that the game ends by time $t$ in the verifiable case and in the unverifiable case, respectively. Then, $S_{V}$ first-order stochastically dominates $S_{U}$.

Proof. From the characterization in Section 4,

$$
\begin{aligned}
S_{V}(t) & =1-e^{-\lambda t} \prod_{i=1}^{2}\left(1-F_{i}(t)\right) \\
& =1-e^{-\lambda t} \prod_{i=1}^{2}\left(1-F_{i}(0)\right) \exp \left(-\left(r \frac{-c+l+p_{j} d}{c}-\lambda\right) t\right)
\end{aligned}
$$

Similarly,

$$
S_{U}(t)=1-e^{-\lambda t} \prod_{i=1}^{2}\left(1-F_{i}(0)\right) \exp \left(-\frac{d-c}{p_{j} d-c}\left(r \frac{-c+l+p_{j} d}{c}-\lambda\right) t\right) .
$$

Since $\frac{d-c}{p_{j} d-c}>1, S_{V}(t)<S_{U}(t)$ for any $t>0$.

This result provides an economic explanation for the payoff equivalence between the two cases in the equilibria with $F_{1}(0)=F_{2}(0)=0$. As explained at the beginning of this section, duplication in information acquisition occurs only with unverifiable information. However, there is less delay with unverifiable information. The gain due to less delay and the loss due to duplication exactly cancel each other out and, thus, players obtain the same payoffs regardless of whether information is verifiable.

\section{Shorter Deadline, Longer Conflict}

This section reports a comparative statics result regarding the arrival rate of public signals, $\lambda .^{13}$ When $\lambda$ increases (the deadline arrives faster), there are two opposing effects. On the one hand, the increase directly speeds up the resolution of the conflict by itself. On the other hand, it indirectly crowds out the players' incentive to acquire information. The following proposition shows that the latter, indirect effect outweighs the former, direct effect, regardless of whether information is verifiable and, thus, the game lasts longer as $\lambda$ increases.

\footnotetext{
${ }^{13}$ Other comparative statics results are rather straightforward. The game last longer when $r$ is smaller, $c$ is larger, or $d$ is larger.
} 
Proposition 6 For any equilibrium, the probabilities that the game ends by time $t$ in each case, $S_{V}(t)$ and $S_{U}(t)$, strictly decrease in $\lambda$.

Proof. From the proof of Proposition 5,

$$
S_{V}(t)=1-e^{\lambda t} \prod_{i=1}^{2}\left(1-F_{i}(0)\right) \exp \left(-r \frac{-c+l+p_{j} d}{c} t\right)
$$

and

$$
S_{U}(t)=1-e^{\left(\frac{d-c}{p_{1} d-c}+\frac{d-c}{p_{2} d-c}-1\right) \lambda t} \prod_{i=1}^{2}\left(1-F_{i}(0)\right) \exp \left(-\frac{d-c}{p_{j} d-c} r \frac{-c+l+p_{j} d}{c} t\right)
$$

As $\lambda$ increases, the marginal benefit of delaying information acquisition increases and, thus, both players are less likely to concede. The decrease of one player's concession rate decreases the other player's marginal benefit. In equilibrium, each player decreases his concession rate by at least as much as the increase in $\lambda$, so that the opponent is again indifferent between acquiring information and waiting. This indirect effect applies to both players and, therefore, is at least twice as large as the direct effect.

It is interesting that the indirect effect is larger when the information is unverifiable. This is because a player's gain when the opponent acquires information is smaller when the information is unverifiable (see Equations (1) and (3)). With verifiable information, a player avoids the information acquisition cost $c$ regardless of whether the game ends due to the arrival of the deadline or due to the opponent's acquisition of information. Therefore, in order to make the opponent remain indifferent, each player needs to reduce his concession rate $\left(\frac{f_{i}(t)}{1-F_{i}(t)}\right)$ by exactly as much as the increase in $\lambda$. With unverifiable information, due to the information rents to the informed player, a player's gain is strictly lower when the game ends due to the opponent's acquisition of information than due to the arrival of the deadline. Therefore, each player must decrease his concession rate by strictly more than the increase in $\lambda$.

\section{Discussion}

The model can be extended in several ways. We discuss two particularly interesting extensions. 


\subsection{Deterministic deadline and fighting cost}

We explain below how our analysis carries over to the case involving a deterministic deadline and fighting cost (no discounting).

\subsubsection{Setup}

We normalize the deadline to be at time $t=1$. The payoff to each player is as follows: for some $k>0$,

$$
U_{i}\left(t_{i}, t_{j}, \omega\right)= \begin{cases}h-k t_{i}, & \text { if }\left(t_{i}>t_{j}\right) \text { or }\left(t_{i}=t_{j}=1 \text { and } \omega=i\right) \\ l-k t_{i}, & \text { if }\left(t_{i}<t_{j}\right) \text { or }\left(t_{i}=t_{j}=1 \text { and } \omega=j\right), \text { or }\left(t_{i}=t_{j}<1\right) .\end{cases}
$$

In words, the winner receives $h$ and the loser receives $l$. Players do not discount future payoffs, but must keep paying a flow cost $k>0$ to stay in the game. The rest of the assumptions and notations are the same as in Sections 2 and 3.

The following two assumptions are analogous to Assumptions 1 and 2 in Section 3.

\section{Assumption 3}

$$
k<p_{i} d, i=1,2 \text {. }
$$

\section{Assumption 4}

$$
c<k \text {. }
$$

Assumption 3 ensures that if no player acquires information, then both players wait until the deadline. Assumption 4 guarantees that players have a non-trivial incentive to acquire information.

\subsubsection{Information acquisition with verifiable information}

As in Section 4, the game is a war of attrition regarding who acquires information first. The analogous equation to Equation (1) is

$$
k=\frac{f_{j}(t)}{1-F_{j}(t)} c
$$

Now the marginal cost of delaying information acquisition is simply the flow fighting cost $k$. Since the deadline is deterministic, the marginal benefit includes only the concession rate of player $j$ times the information-acquisition cost.

Using the equation, we can construct a continuum of equilibria, as in Section 4. The following proposition is parallel to Proposition 2. 
Proposition 7 When acquired information is verifiable, there is a continuum of equilibria: for any $F_{1}(0), F_{2}(0) \in[0,1]$ such that $F_{1}(0) F_{2}(0)=0$, there is an equilibrium in which each player acquires information according to a distribution function $F_{i}(t)=1-$ $\left(1-F_{i}(0)\right) \exp \left(-\frac{k}{c} t\right)$ over time $[0, \bar{t}]$, where $\bar{t}=1-\frac{c}{k}$, and simply waits until the deadline after $\bar{t}$. The set of equilibrium payoffs is given by

$\left\{\left(v_{1}, v_{2}\right): v_{i} \in\left[-c+l+p_{i} d, l+p_{i} d\right]\right.$, and $\left.\left(v_{1}-\left(-c+l+p_{1} d\right)\right)\left(v_{2}-\left(-c+l+p_{2} d\right)\right)=0\right\}$.

The only essential difference from Proposition 2 is the presence of $\bar{t}$, which is the last time players may acquire information in the equilibrium. Such $\bar{t}$ exists because when it is sufficiently close to the deadline, players strictly prefer waiting until the deadline to incurring the information-acquisition cost. $\bar{t}$ is the time at which players are indifferent between ending the game immediately by acquiring information and waiting until the deadline, that is, $-c+l+p_{i} d=-(1-\bar{t}) k+l+p_{i} d$.

\subsubsection{Information acquisition with unverifiable information}

Unless it is close to the deadline (precisely, before $1-\frac{c}{k}$ ), the subgame outcome where only one player is informed is exactly the same as in Section 5 . The weak informed player concedes with probability $1-\frac{p_{i} c}{p_{j}(d-c)}$ immediately. If the informed player does not concede, then the uninformed player acquires information with probability 1 , but he is indifferent between acquiring information and conceding.

In the original game, the analogous condition to Equation (3) is

$$
k=\frac{f_{j}(t)}{1-F_{j}(t)} \frac{p_{i} d-c}{d-c} c .
$$

The usual interpretations apply to each side. The following proposition corresponds to Proposition 4.

Proposition 8 When acquired information is unverifiable, there is a continuum of equilibria: for any $F_{1}(0), F_{2}(0) \in[0,1]$ such that $F_{1}(0) F_{2}(0)=0$, it is an equilibrium that each player acquires information according to a distribution function $F_{i}(t)=1-(1-$ $\left.F_{i}(0)\right) \exp \left(-\frac{k}{c} \frac{d-c}{p_{i} d-c} t\right)$ over time $[0, \bar{t}]$, where $\bar{t}=1-\frac{c}{k}$, and simply waits until the deadline after $\bar{t}$. The set of equilibrium payoffs is given by

$$
\left\{\left(v_{1}, v_{2}\right): v_{i} \in\left[-c+l+p_{i} d, l+\frac{p_{i} d-c}{d-c} d\right], \text { and }\left(v_{1}-\left(-c+l+p_{1} d\right)\right)\left(v_{2}-\left(-c+l+p_{2} d\right)\right)=0\right\} .
$$




\subsection{Unobservable information acquisition}

We assumed that if a player acquires information, then the opponent knows that the player is informed about the state, regardless of whether information is verifiable. The assumption is innocuous for the verifiable-information case, but not for the unverifiable-information case.

Suppose the information is verifiable, but a player's information acquisition is not observable by the opponent. Pick any equilibrium in Proposition 2 and consider the following strategy profile: each player acquires information according to $F_{i}$ and discloses the acquired information regardless of its content (or, he simply concedes if the state is unfavorable to him). In this strategy profile, the only potential incentive problem is whether a weak player would be willing to concede immediately. But given that the opponent would concede only when he himself finds out that the state is unfavorable to him, a weak player strictly prefers conceding immediately. Therefore, any equilibrium outcome in Proposition 2 can be supported as an equilibrium outcome even when information acquisition is not observable.

When information is not verifiable, no equilibrium outcome in Proposition 4 can be supported as an equilibrium outcome with unobservable information acquisition. This is because the subgame outcome after one player's acquisition of information dramatically changes, since, by definition, the opponent does not know that the other player is informed. It is not obvious whether the players would acquire information faster (and whether the game would last longer). A players would have a weaker direct incentive to acquire information, because even if he knows that the state was favorable to him, he would have to bear some delay costs. On the other hand, the fact that the opponent would acquire information more slowly implies that players would have a lower marginal benefit from delaying information acquisition and, thus, players would have a stronger indirect incentive to acquire information.

We expect that if the information is unverifiable, then the players' expected payoffs would be lower when information acquisition is not observable ${ }^{14}$ and a typical equilibrium would consist of two regimes: first, players would gradually acquire information and, second, once uninformed players become sufficiently pessimistic, they would play a standard war of attrition with incomplete information. This case is theoretically interesting. Not only does the symmetric-information game turn into a game with asymmetric information at some point, but also the players face a complicated inference problem. They must constantly update their beliefs about the opponent's status (whether he has acquired information and, if so, whether he received a favorable information) as well as infer what beliefs the opponent

\footnotetext{
${ }^{14}$ We know that this is true for $c$ rather large. Precisely, if (and only if) $c \geq \frac{r}{r+\lambda} p_{1} l$ (this inequality does not violate Assumption 2), then there is an equilibrium in which no player acquires information: player $i$ 's equilibrium payoff is $\frac{\lambda}{r+\lambda}\left(p_{i} h+\left(1-p_{i}\right) l\right)$, which is larger than his deviation payoff by acquiring information $-c+p_{i} \frac{\lambda}{r+\lambda} h+\left(1-p_{i}\right) l$ whenever $c \geq \frac{r}{r+\lambda} p_{i} l$.
} 
would have about his beliefs in each status. We leave this challenging problem for future research.

\section{Appendix}

\section{Proof of Proposition 3:}

(1) Weak player $i$ obtains only $l$.

Suppose not. It can happen only when player $j$ concedes without acquiring information with a certain probability. Therefore, player $j$ 's expected payoff must be equal to $l$. But since weak player $i$ would not quit immediately, player $j$ 's belief over the true state would not change, which implies that player $j$ can secure $-c+l+p_{j} d$ by acquiring information. By Assumptions 1 and 2, the latter payoff is strictly larger than the former and, thus, this is a contradiction.

(2) At the beginning of the subgame, weak player $i$ randomizes between conceding and waiting.

The argument given before the proposition applies.

(3) Let $\alpha$ be the probability that weak player $i$ concedes at the beginning of the subgame. An instant later, player $j$ must be indifferent between acquiring information and conceding without acquiring information. Therefore,

$$
\alpha=\frac{p_{j} d-c}{p_{j}(d-c)} .
$$

Suppose player $j$ strictly prefers acquiring information to conceding. Then weak player $i$ obtains only $l$, regardless of whether player $j$ acquires information or waits for a public signal. But then an instant before (at the beginning of the subgame), weak player $i$ strictly prefers conceding to waiting, which contradicts (2). Now suppose player $j$ strictly prefers conceding to acquiring information. For this and (2) to be simultaneously true, both weak player $i$ and player $j$ must be indifferent between conceding and waiting for a public signal. This implies that, again, weak player $i$ obtains only $l$, which creates the same contradiction as the previous case.

(4) An instant after the beginning of the subgame, player $j$ either acquires information or concedes. That is, player $j$ does not simply wait for a public signal.

Suppose player $j$ strictly prefers waiting to conceding. For (2) to be true, weak player $i$ must strictly prefer waiting to conceding as well (otherwise, weak player $i$ must prefer conceding earlier). The latter implies that player $j$ 's belief does not change at the next instant and, thus, he again strictly prefers waiting to conceding or acquiring information. In 
addition, by the same argument as above, weak player $i$ does not concede. This process will continue until a public signal arrives. But weak player $i$ 's expected payoff is then strictly smaller than $l$, which contradicts (4).

Now suppose player $j$ is indifferent among waiting, conceding, and acquiring information and he waits with a positive probability. Similarly to the previous case, weak player $i$ must strictly prefer waiting to conceding. He also must strictly prefer waiting to conceding at the next instant as well, because otherwise, at the following instant, player $j$ 's belief would decrease, player $j$ would concede immediately, and then weak player $i$ would obtain strictly more than $l$, which would contradict (2). This process will continue until player $j$ acquires information, player $j$ concedes, or a public signal arrives. Suppose player $j$ waits for a signal forever. Since weak player $i$ never concedes, player $j$ 's expected payoff is

$$
\frac{\lambda}{r+\lambda}\left(l+\frac{p_{j}(1-\alpha)}{p_{i}+p_{j}(1-\alpha)} d\right)=\frac{\lambda}{r+\lambda}(l+c) .
$$

Under Assumptions 1 and 2, this payoff is strictly smaller than $l$, which contradicts the fact that player $j$ is indifferent between waiting and conceding.

(5) Player $j$ acquires information with probability 1 , that is, player $j$ concedes with negligible probability.

Otherwise, then weak player $i$ would strictly prefer waiting an instant to conceding immediately, which would contradict (2). As shown in the main content, in discrete time, player $j$ concedes with a positive probability, but the probability approaches zero as $\Delta$ tends to zero. Q.E.D.

\section{References}

Bishop, D.T. and C. Cannings (1978), 'A Generalized War of Attrition', Journal of Theoretical Biology, 70, 85-124.

Bishop, D.T., C. Cannings, and J. Maynard Smith (1978), 'The War of Attrition with Random Rewards', Journal of Theoretical Biology, 74, 377-388.

Bolton, P. and C. Harris (1999), 'Strategic Experimentation', Econometrica, 67, 349-374.

Bulow, J. and P. Klemperer (1999), 'The Generalized War of Attrition', American Economic Review, 89(1), 175-189.

Burdett, K., S. Shi, and R. Wright (2001), 'Pricing and Matching with Frictions', Journal of Political Economy, 109(5), 1060-1085. 
Crémer, J. and F. Khalil (1992), 'Gathering Information Before Signing a Contract', American Economic Review, 82, 566-578.

Crémer, J., F. Khalil, and J.C. Rochet (1998a), 'Contracts and Productive Information Gathering', Games and Economic Behavior, 25, 174-193.

Crémer, J., F. Khalil, and J.C. Rochet (1998b), 'Strategic Information Gathering before a Contract Is Offered', Journal of Economic Theory, 81, 163-200.

Chatterjee, K. and W. Samuelson (1987), 'Bargaining with Two-Sided Incomplete Information', Review of Economic Studies, 54, 175-192.

Dang, T. (2007), 'Information Acquisitions in Double Auctions', Working Paper, University of Mannheim.

Dang, T. (2008), 'Bargaining with Endogenous Information', Journal of Economic Theory, 140, 339-354.

Ely, J. and J. Välimäki (2003), 'Bad Reputation', Quarterly Journal of Economics, 118, 785814.

Fershtman, C. and D. Seidmann (1993), 'Deadline Effects and Inefficient Delay in Bargaining with Endogenous Commitment', Journal of Economic Theory, 60, 306-321.

Fudenberg, D. and J. Tirole (1986), 'A Theory of Exit in Duopoly', Econometrica, 54, 943960.

Fudenberg, D., R. Gilbert, J. Stiglitz, and J. Tirole (1983), 'Preemption, Leapfrogging and Competition in Patent Races', European Economic Review, 22, 3-31.

Ghemawat, R. and B. Nalebuff (1985), 'Exit', Rand Journal of Economics, 16, 184-194.

Grossman, S.J. (1981), 'The Informational Role of Warranties and Private Disclosure about Product Quality', Journal of Law and Economics, 24(3), 461-483.

Grossman, S. and J. Stiglitz (1980), 'On the Impossibility of Informationally Efficient Markets', American Economic Review, 70, 393-408.

Hendricks, K., A. Weiss and C. Wilson (1988), 'The War of Attrition in Continuous Time with Complete Information', International Economic Review, 29, 663-680.

Jansen, J. (2008), 'Information Acquisition and Strategic Disclosure in Oligopoly', Journal of Economics 83 Management Strategy, 17(1), 113-148. 
Keller, G., S. Rady, and M. Cripps (2005), 'Strategic Experimentation with Exponential Bandits', Econometrica, 73(1), 39-68.

Kreps, D. and R. Wilson (1982), 'Reputation and Imperfect Information', Journal of Economic Theory, 27, 253-279.

Levin, D. and J.L. Smith (1984), 'Equilibrium in Auctions with Entry', American Economic Review, 84(3), 585-599.

Li, H. (2001), 'A Theory of Conservatism', Journal of Political Economy, 109(3), 617-636.

Liu, Q. (2010), 'Informaiton Acquisition and Reputation Dynamics', Review of Economic Studies, forthcoming.

Ma, C. and M. Manove (1993), 'Bargaining with Deadlines and Imperfect Player Control', Econometrica, 61(6), 1313-1339.

Maynard Smith, J. (1974), 'The Theory of Games and the Evolution of Animal Conflicts', Journal of Theoretical Biology, 47, 209-221.

Matthews, S. (1984), 'Information Acquisition in Discriminatory Auctions', in: Boyer, M., Kihlstrom, R.E. (eds), Bayesian Models in Economic Theory, 181-207.

Milgrom, P. R. (1981), 'Good News and Bad News: Representation Theorems and Applications', Bell Journal of Economics, 12(2), 380-391.

Milgrom, P. R. and R. Weber (1985), 'Distributional Strategies for Games with Incomplete Information', Mathematics of Operations Research, 10(2), 619-632.

Morath, F. (2010), 'Volunteering and the Strategic Value of Ignorance', WZB Discussion Paper No. SP II 2010-17.

Morath, F. and J. Münster (2010), 'Information Acquisition in Conflicts', STB/TR 15 Discussion Paper No. 314.

Nalebuff, B. and J. Riley (1985), 'Asymmetric Equilibria in the War of Attrition', Journal of Theoretical Biology, 113, 517-527.

Ordover, J., A. Rubinstein (1986), 'A Sequential Concession Game with Asymmetric Information', Quarterly Journal of Economics, 101, 879-888.

Persico, N. (2000), 'Information Acquisition in Auctions', Econometrica, 68, 135-148. 
Persico, N. (2004), 'Committee Design with Endogenous Information', Review of Economic Studies, 71(1), 165-191.

Ponsati, C. (1995), 'Deadline Effect: A Theoretical Note', Economic Letters, 48, 281-285.

Ponsati, C. and J. Sákovics (1995), 'The War of Attrition with Incomplete Information', Mathematical Social Sciences, 29, 239-254.

Riley, J. (1980), 'Strong Evolutionary Equilibrium and the War of Attrition', Journal of Theoretical Biology, 83, 383-402.

Shavell, S. (1994), 'Acquisition and Disclosure of Information Prior to Sale', Rand Journal of Economics, 25, 20-36.

Spier, K. (1992), 'The Dynamics of Pre-trial Negotiation', Review of Economic Studies, 59, 93-108. 